\title{
LOD2 BUILDING GENERATION EXPERIENCES AND COMPARISONS
}

\author{
Iuliana Maria Pârvu ${ }^{\text {a, }} *$, F. Remondino ${ }^{\text {b }}$, E. Ozdemir ${ }^{\text {b }}$ \\ a* National Center of Cartography, Cartography and Photogrammetry Department, 012101; Doctoral School, Technical University \\ of Civil Engineering of Bucharest, 020396, Romania, e-mail: iuliana.parvu@cngcft.ro \\ b Bruno Kessler Foundation (FBK), 38123, Italy, e-mails: remondino@ fbk.eu, eozdemir@ fbk.eu
}

Received: 14.10.2018 / Accepted: 12.11.2018/ Revised: 20.11.2018 / Available online: 15.12.2018

DOI: 10.2478/jaes-2018-0019

KEY WORDS: point cloud, segmentation, region growing, digital terrain model, digital surface model

\begin{abstract}
:
The VOLTA project is a RISE Marie-Curie action designed to realize Research \& Innovation (R\&I) among intersectoral partners to exchange knowledge, methods and workflows in the geospatial field. To accomplish its objectives, the main R\&I activities of VOLTA are divided in four interlinked Work Packages with two transversal ones responsible for knowledge transfer \& training as well as dissemination of the project results. The research activities and knowledge transfer are performed with a series of secondments between partners. The consortium is composed of 13 partners from academic \& research institutions, industrial partners and national mapping agencies. The Romanian National Center of Cartography is part of this research project and in this article the achievements of the secondment at Bruno Kessler Foundation in Trento (Italy) are given. The main goal of the exchange was to generate level of detail - LOD2 building models in an automated manner from photogrammetric point clouds and without any ancillary data. To benchmark existing commercial solutions for the realization of LOD2 building models, we tested Building Reconstruction. This program generates LOD2 models starting from building footprints, digital terrain model (DTM) and digital surface model (DSM). The presented work examined a research and a commercial-based approach to reconstruct LOD2 building models from point clouds. The full paper will report all technical details of the work with insight analyses and comparisons.
\end{abstract}

\section{INTRODUCTION}

3D city models are digital models of urban areas that represent terrain surfaces, sites, buildings, vegetation, infrastructure, city furniture etc. They are becoming an important tool in modern days, they are used in city planning and environmental simulations, such as energy estimation, solar potential, shadow analysis, emergency management, property management etc.

In order to create a 3D city model, it is important to have tools and skills for extracting individual buildings from the collected data. 3D models differ a lot, according on the acquisition and processing methods. In this paper, the generation of 3D building models using data from the International Society for Photogrammetry and Remote Sensing (ISPRS) benchmark on oblique imagery is presented.

The concept of LOD is defined by the Open Geospatial Consortium, Inc. and is one of the most important characteristics of a 3D city model. The LOD of a 3D model (Figure 1) gives the geometric details of it and was developed for several thematic classes of objects but is used the most for buildings (Biljecki et al., 2016). There are five types of LODs in buildings, in this paper we will focus our attention on LOD2, i.e. a model with a simplified roof shape, where the object's

parts can be modelled in multiple semantic classes (e.g. roof, walls).
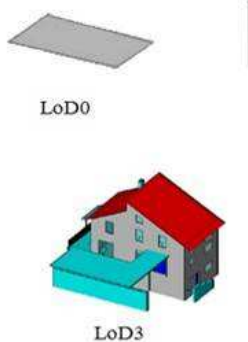

Figure 1. Levels of detail (Gröger \& Plümer, 2012)

The information needed to generate 3D building models are normally derived from point clouds, acquired or with Light Detection and Ranging (LiDAR) sensors, or derived from photogrammetric images, particularly obtained from oblique cameras.

\footnotetext{
* Corresponding author: Iuliana Maria PÂRVU, e-mail: iuliana.parvu@cngcft.ro
} 
Oblique cameras (Remondino \& Gerke, 2015) were used during World War I and World War II, but this kind of system was very expensive at that time. After the digital period began, oblique cameras were reinserted on the photogrammetric market. The most recent oblique camera system uses Maltesecross configuration (Figure 2), with five cameras: one for nadir views and four for the cardinal views.

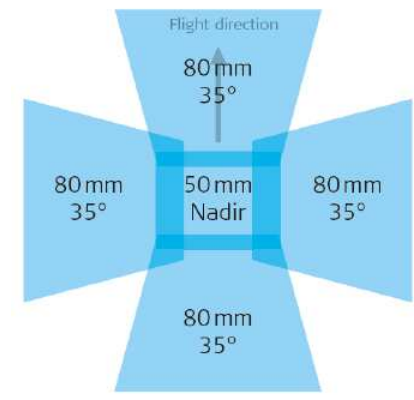

Figure 2. Example of Maltese cross configuration in airborne oblique cameras (Remondino \& Gerke, 2015)

The number of images acquired using oblique technology is much bigger then in traditional photogrammetry, so there is a huge amount of data to be managed.

Some of the advantages of oblique cameras are: the visibility of roofs and vertical structures (facades), a more simpler and better interpretation of urban areas (visibility of building footprints, possibility of inspecting building heights, counting floors or checking changes on the facades), the generation of $3 \mathrm{D}$ point clouds, etc. The most important disadvantages of oblique imagery lay in the data processing, including tie point extraction, dense image matching, etc.

National Mapping Agencies (NMAs), such as the Romanian National Center of Cartography (CNC), see the potential of oblique imagery in updating and managing cadaster information and are willing to make products based on oblique imagery in the near future (Remondino et al., 2016).

There are many approaches presented in the literature for $3 \mathrm{D}$ building modelling, which rely on point clouds (Haala \& Kada, 2010; Lafarge \& Mallet, 2012 etc.). Dorninger and Pfeifer developed a workflow based on the following steps: building detection, extraction of approximate contours, roof reconstruction, 3D model generation and assessment of the model (Dorninger \& Pfeifer, 2008). But most of this methods require ancillary data such as building footprints. In most cases, reliable footprints are not available. In this paper we will focus on developing a workflow for generating 3D building models without relying on ancillary data and this topic will be presented in chapter 3. Another approach is to reconstruct 3D building models using building footprints (chapter 4).

\section{VOLTA PROJECT}

The VOLTA project (http://volta.fbk.eu) is a RISE Marie-Curie action designed to realize research and innovation among partners to exchange knowledge, methods and workflows in the geospatial field. The research activities and knowledge transfer are performed with a series of secondments between partners.
This project plans to design and validate innovative processing and handling methodologies for 3D geospatial data to support practical applications of photogrammetric mapping approaches.

The novelty that VOLTA brings is manifold:

- reduce the gap between research and development partners and the public mapping sector to transfer useful methodologies and practices;

- $\quad$ exchange workflows and technological solutions among companies and researchers to improve scientific expertise and service capabilities;

- advance the development of Spatial Data Infrastructure through stronger intersectoral collaboration among geospatial players.

The main R\&I activities of VOLTA are divided in four interlinked Work Packages (WPs - Figure 3) with two transversal ones responsible for knowledge transfer and training as well as dissemination of the project results.

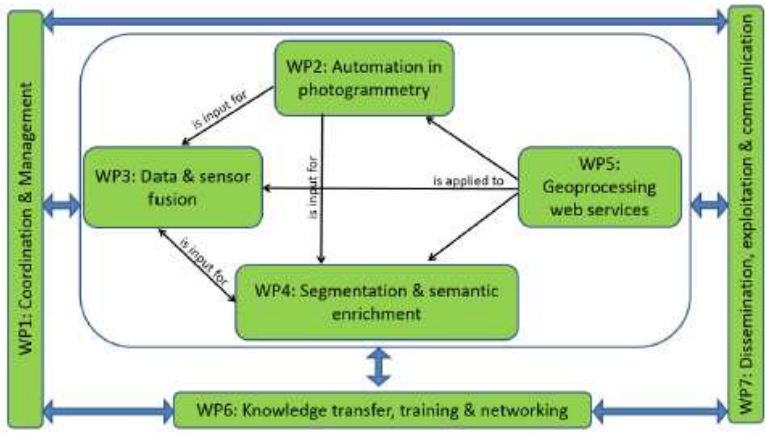

Figure 3. VOLTA WPs (REA Grant Agreement, 2016)

The consortium is a well-balanced team, composed of 13 partners from 11 countries, divided in academic (Technische Universität Wien, Gottfried Wilhelm Leibniz Universität Hannover, Uniwersytet Warszawski, Technische Universiteit Delft) and research (Bruno Kessler Foundation) institutions, industrial partners (Geodetski Zavod Celje, Vermessung AVT, VEXCEL Imaging, Geoimaging) and national mapping and cartographic agencies (Institut National De L'information geographique Et Forestiere, Ordnance Survey, Institut Cartogràfic I Geològic De Catalunya, CNC).

VOLTA is aligned with various (closed or on-going) national and international geospatial projects. These projects include recently funded EU H2020 actions and other projects such as: EuroGlobalMap (provides and maintains a seamless and harmonized topographic digital data set at scale 1:1.000.000), Copernicus (the European system for monitoring the Earth by collecting data from multiple sources, processing them and providing users with reliable and up-to-date information), GeoKnow (an EU funded project for linking geospatial data from heterogeneous information systems and uses the web as a platform to exploit these data) etc.

As shown in Figure 3, there are four technical WPs, which will bring innovation in our field of activity, as follows:

- WP 2 - Automation in photogrammetry. The WP focuses on gathering metric $3 \mathrm{D}$ information from satellites, airborne sensors, unmanned aerial vehicles (UAV) and terrestrial image data. The WP will innovate 
existing automated image processing methods, from image orientation to dense matching, for improving production pipelines;

- WP 3 - Data \& sensor integration. This WP is working with the integration of various sensory data in a convergence-of-evidence approach, whose aim is to take advantage of the unique features of each and overcome their shortcomings;

- WP 4 - Segmentation \& semantic enrichment of geospatial data. In this WP will be developed and implemented new classification and segmentation solutions in operating mode for images and point clouds;

- WP 5 - Geoprocessing web services. The WP will consider the deployment and parallelization as webservice in the Cloud of the geospatial frameworks, algorithms and methodologies developed within the previous WPs (REA Grant Agreement, 2016).

In this paper, the focus will be on a secondment in VOLTA project on the topic 3D building modelling, from WP2.

\section{RESEARCH APPROACH FOR LOD2 BUILDING MODEL GENERATION}

The goal of the research was to generate LOD2 building models in an automated manner from photogrammetric point clouds, without using any ancillary data. The tests were performed using the ISPRS benchmark on oblique imagery (Nex et al., 2015). A typical way to obtain LOD2 buildings models is to (i) segment the available point cloud, (ii) identify the roof areas, (iii) define planar surfaces and (iv) generate 3D building geometries.

The point cloud segmentation was performed using a FBK tool for the region growing algorithm based on Point Cloud Library (Ozdemir \& Remondino, 2018). The purpose of the algorithm is to merge the points that are close enough in terms of the smoothness constraint (Rabbani et al., 2006). Thereby, the output of this algorithm is a set of clusters, were each cluster consists of a set of points that are considered to be a part of the same smooth surface. The work of this algorithm is based on the comparison of the angles between the normal of the points and curvature values (http://pointclouds.org/documentation/tutorials /region_growing_segmentation.php). So, normal computation is a very important step in the process. When computing normal on a point cloud, it is necessary to estimate the local surface represented by a point and its neighbours. The level of noise and the number of neighbours will change how this surface looks like.

The region growing algorithm is shown in Figure 4. First of all, the algorithm sorts the points by their curvature value, to begin the region growing from the point that has the minimum curvature value (this point becomes the first seed). For every seed, the algorithm identifies the neighbours and computes the angles between the normal of the neighbour and the normal of the seed. If the angle for a specified point is less than the threshold value, that point is added to the current cluster. After this step is finished, every neighbour is tested for the curvature value. If the curvature is less than the threshold value then the point is added to the seeds.

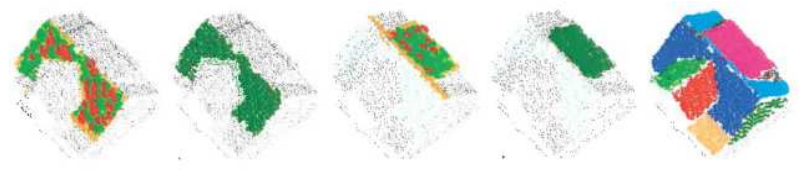

Figure 4. Examples of region growing algorithm (Dorninger \& Pfeifer, 2008)

If the seeds set becomes empty it means that the algorithm has grown the region and the process is repeated from the beginning. This algorithm uses the following parameters:

- K Search: number of points that defines the local surface used for computing the normals;

- Min Cluster Size: minimum size of the cluster;

- Max Cluster Size: maximum size of the cluster;

- Number Of Neighbours: number of neighbours;

- Smoothness Threshold: threshold set for the normal deviation;

- Curvature Threshold: threshold set for curvature value.

In the following, some of the tests made on the dataset are shown. The goal was to extract each building in a separate cluster and to decrease the number of eliminated points.

\subsection{Tests for the Dortmund City Hall}

Table 1. Parameters and results for the region growing

\begin{tabular}{|c|c|c|c|c|c|c|c|}
\hline \multirow{2}{*}{ PARAM. } & \multicolumn{7}{|c|}{ TESTS } \\
\cline { 2 - 8 } & 1 & 2 & 3 & 4 & 5 & 6 & 7 \\
\hline K Search & 50 & 50 & 50 & 50 & 50 & 50 & 50 \\
\hline $\begin{array}{c}\text { Min Cluster } \\
\text { Size }\end{array}$ & 250 & 50 & 250 & 250 & 250 & 250 & 250 \\
\hline $\begin{array}{c}\text { Max Cluster } \\
\text { Size (MIIL) }\end{array}$ & 10 & 10 & 10 & 10 & 10 & 10 & 10 \\
\hline $\begin{array}{c}\text { Number Of } \\
\text { Neighbours }\end{array}$ & 200 & 200 & 50 & 150 & 200 & 200 & 200 \\
\hline $\begin{array}{c}\text { Smothothness } \\
\text { Threshold }\end{array}$ & 3 & 3 & 3 & 3 & 5 & 2.5 & 3.25 \\
\hline $\begin{array}{c}\text { Curvature } \\
\text { Threshold }\end{array}$ & 1.15 & 1.15 & 1.15 & 1.15 & 1.15 & 1.15 & 0.85 \\
\hline $\begin{array}{c}\text { NUMBER } \\
\text { OF } \\
\text { CLUUSTERS }\end{array}$ & 69 & 269 & 93 & 77 & 35 & 74 & 63 \\
\hline $\begin{array}{c}\text { ELIM. } \\
\text { POINTS (\%) }\end{array}$ & 23 & 20 & 40 & 26 & 9 & 30 & 20 \\
\hline
\end{tabular}

As shown in Table 1, if we decrease the minimum cluster size, the number of clusters grows. Based on this fact, we used for our dataset the minimum size of the cluster - 250. Even if, for TEST 5 (Figure 5d), the number of clusters and the percentage of eliminated points is smaller, this is not the best combination because the ground is in the same cluster as parts of the building. The best result is obtained for TEST 7 (Figure 5f).
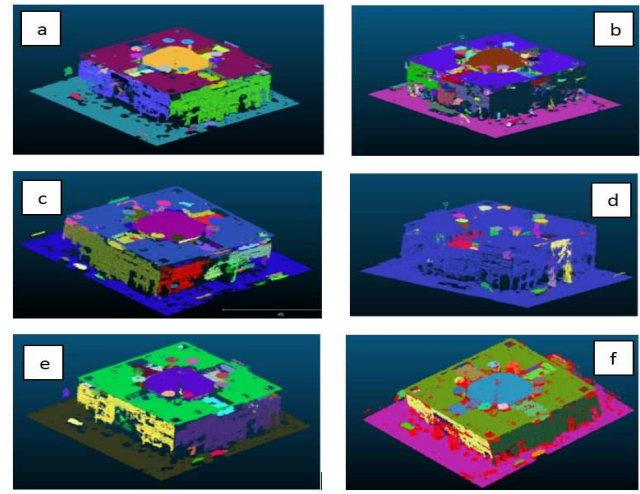

Figure 5. Clusters after segmentation for the City Hall building based on parameters from Table 1 (a. TEST 1, b. TEST 2, c. TEST 4, d. TEST 5, e. TEST 6, f. TEST 7) 
Another important aspect is to decrease the number of eliminated points, examples of the results are shown in Figure 6.
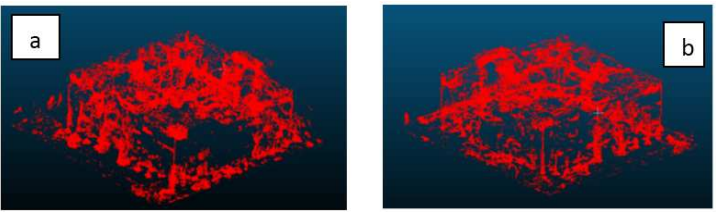

Figure 6. Points eliminated after segmentation for the City Hall building ( $a$. TEST 1, b. TEST 2)

\subsection{Tests for an area in the city of Dortmund}

In Table 2 are displayed the parameters used and the results obtained after segmentation for an area the city of Dortmund.

Table 2. Parameters and results for the region growing

\begin{tabular}{|c|c|c|c|c|c|c|c|}
\hline \multirow{2}{*}{ PARAM. } & \multicolumn{7}{|c|}{ TESTS } \\
\cline { 2 - 8 } & 1 & 2 & 3 & 4 & 5 & 6 & 7 \\
\hline K Search & 50 & 50 & 50 & 50 & 100 & 200 & 300 \\
\hline $\begin{array}{c}\text { Min Cluster } \\
\text { Size }\end{array}$ & 2500 & 2500 & 2500 & 1000 & 500 & 500 & 500 \\
\hline $\begin{array}{c}\text { Max } \\
\text { Cluster Size } \\
\text { (MIL) }\end{array}$ & 10 & 10 & 10 & 10 & 1 & 1 & 1 \\
\hline $\begin{array}{c}\text { Number Of } \\
\text { Neighbours }\end{array}$ & 100 & 500 & 150 & 150 & 150 & 150 & 150 \\
\hline $\begin{array}{c}\text { Smoothness } \\
\text { Threshold }\end{array}$ & 3.25 & 3.25 & 3.25 & 3.25 & 3.25 & 3.25 & 3.25 \\
\hline $\begin{array}{c}\text { Curvature } \\
\text { Threshold }\end{array}$ & 1.15 & 1.15 & 1.15 & 1.15 & 1.15 & 1.15 & 1.15 \\
\hline $\begin{array}{c}\text { NUMBER } \\
\text { OF }\end{array}$ & 94 & 12 & 82 & 144 & 286 & 304 & 267 \\
CLUSTERS & 74 & 38 & 64 & 61 & 51 & 39 & 31 \\
\hline $\begin{array}{c}\text { ELIM. } \\
\text { POINTS (\%) }\end{array}$ & 74 & \multicolumn{7}{|c|}{} \\
\hline
\end{tabular}
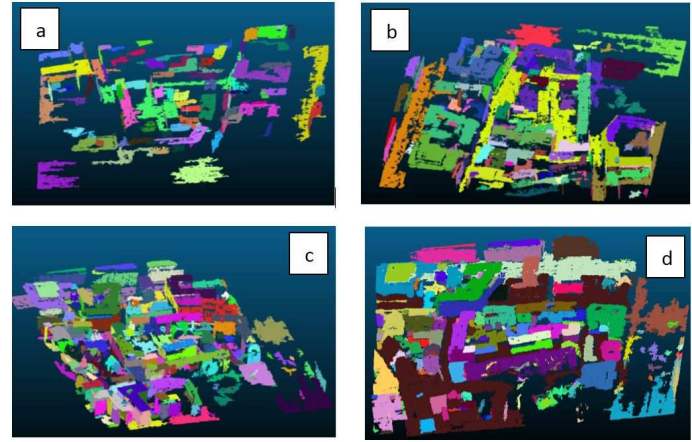

Figure 7. Clusters after segmentation in Dortmund based on parameters from Table 2

(a. TEST 1, b. TEST 3, c. TEST 5, d. TEST 7)

The best results are obtained with the parameters from TEST 7 (Figure 7). In the end, the point cloud is splinted in as many point clouds as the number of clusters. The next step is to extract the primitives using Random sample consensus (RANSAC) method in Mapple (Figure 8) and then to generate the $3 \mathrm{D}$ building model for each building in Polyfit software (https://3d.bk.tudelft.nl/liangliang/software.html).

RANSAC is an iterative method to estimate parameters of a mathematical model from a set of observed data that contains outliers. It is a non-deterministic algorithm, it produces a reasonable result only with a certain probability, with this probability increasing as more iterations are allowed (https://en.wikipedia.org/wiki/Random_sample_consensus).

An example of planar segments using this method is displayed in Figure 9. The higher the number of primitives, the higher the processing time will be. The primitives are saved in a text file (.vg format) that contains information for every point (coordinates and computed normal) and data for every plane formed.

Polyfit is based on hypothesizing and selection strategy. In the first step an optimal set of faces from a large number of candidate faces is chosen to assemble a compact polygonal surface model. The optimal set of faces is selected through optimization that encourages good point support and compactness of the final model (Nan \& Wonka, 2017). In the optimization step, there can be used one of the following solvers: SCIP, GUROBI, GLPK and LPSOLVE. For Dortmund City Hall were used two solvers SCIP and LPSOLVE to generate the $3 \mathrm{D}$ building model and the best result was obtained using LPSOLVE (Figure 10).

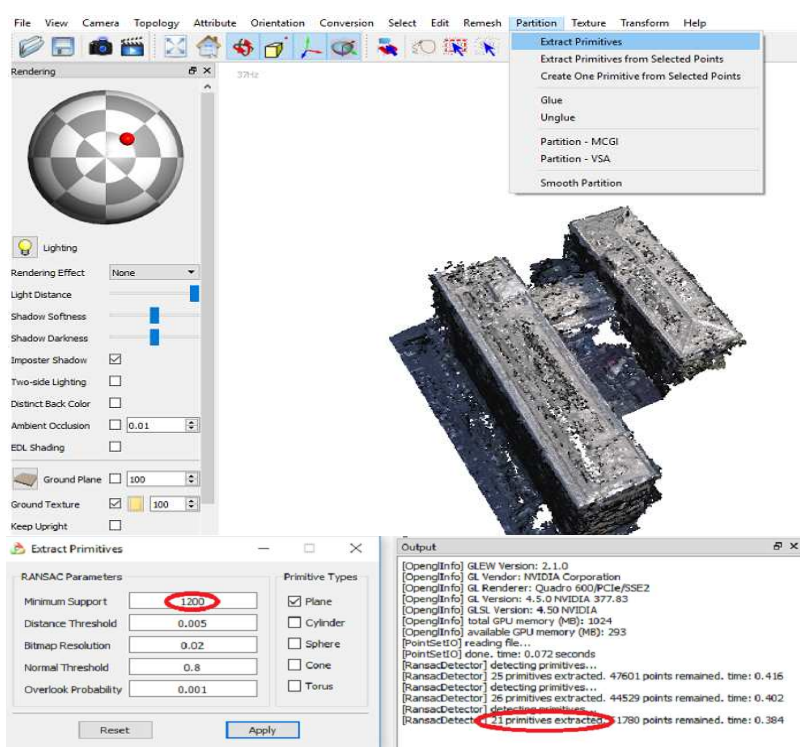

Figure 8. A view of Mapple software

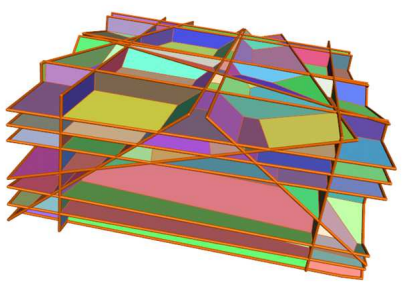

Figure 9. Planar segments using RANSAC

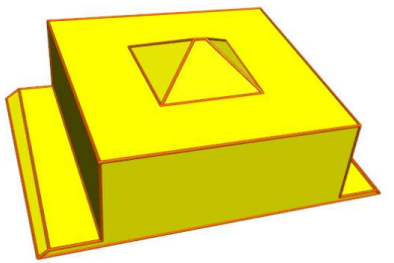

Figure 10. 3D building model of the City Hall (LPSOLVE) 


\section{COMMERCIAL-BASED APPROACH FOR LOD2 BUILDING MODELS GENERATION}

To benchmark existing commercial solutions for the realization of LOD2 building models, we tested the software Building Reconstruction, developed by Virtual City System (2018). Mandatory input data are:

- DSM - resolution between $50 \mathrm{~cm}$ and $20 \mathrm{~cm}$, in ASCII grid format or as point cloud (a better resolution will not improve the result);

- DTM - resolution of $1 \mathrm{~m}$, in ASCII grid format or as point cloud;

- Building footprints - closed polygons with unique identifier.

For the final visual check of the derived $3 \mathrm{D}$ geometries it is possible to use digital orthoimages, with a resolution of at least $40 \mathrm{~cm}$ or a shaded DSM. These files facilitate the quality control work.

Before starting the reconstruction process the dataset must be divided in tiles of $1 \times 1 \mathrm{~km}$. A tiling software is needed for this operation. The ground information taken from the DTM is used to determine the ground level of the buildings. This fact guarantees that buildings will stay perfectly on the ground. The DSM is used to determine the building height and extract primitives for the roof areas, using algorithms such as rectangle decomposition, cell decomposition and extrusion. The process ensures that the roofs do not exceed the footprint of buildings and thus provides compliance with the $2 \mathrm{D}$ map.

The pipeline can be customized based on a set of parameters (e.g. to ignore the buildings with an area under a specified value or to set flat roof for buildings under a specified area value). Complex buildings cannot be reconstructed accordingly to their real shape, so they must be treated separately, in a manual way. If the density of the point cloud is at least 4 points/square meters, the height accuracy is under 0.5 meters. The positional accuracy for buildings is $100 \%$ based on the accuracy of the footprints. The software provides a report that contains the total number of buildings, the number of unprocessed and omitted buildings, the automatically reconstructed buildings, the modified buildings, the buildings with default height, the reconstruction method, the average number of polygons per building, the buildings per quality class (https://www.virtualcitysystems.de/en/).

The tool was tested on three different sets of point clouds: two obtained from oblique imagery over Imst (Austria) and Bergamo (Italy) and one obtained using LiDAR aerial scanning over Săcuieni (Romania).

For Bergamo, being the medieval part of the city, the process did not achieve successful 3D results, probably due to the complexity of the buildings and the large number of vertices in the building footprint data.

For Imst, the process achieved reasonable results only after generalizing the building footprints and resolving the topological errors.

For the Săcuieni dataset, the process achieved good results (Figure 11, 12, 13) and the generated buildings have semantic information attached, such as: roof type, highest vertex for the roof, ground area.

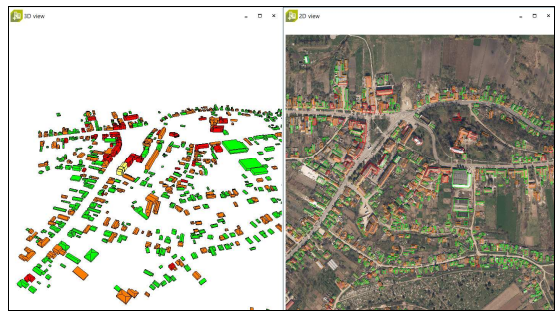

Figure 11. LOD2 3D building models for Săcuieni (Romania)

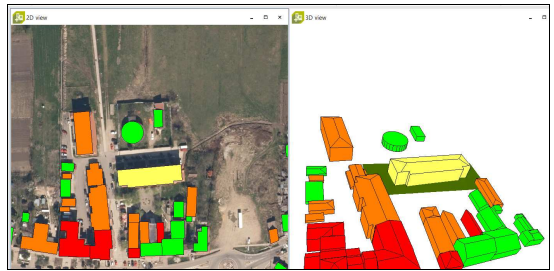

Figure 12. 3D building models for a tile in Săcuieni City

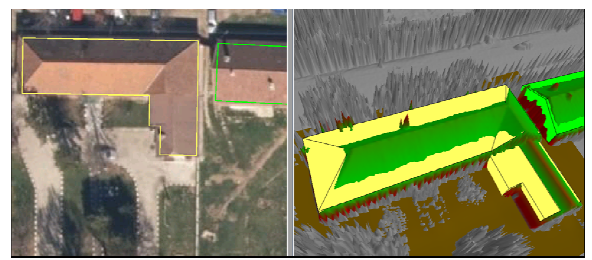

Figure 13. Building footprint and 3D building model

For the case study, 708 buildings were reconstructed, 682 by cell decomposition and 26 by extrusion.

The generated 3D buildings were divided in three quality classes (Table 3), based on the value of the standard deviation (STDEV) (Figure 14).

Table 3. Quality classes

\begin{tabular}{|c|c|c|}
\hline Quality Class & STDEV (m) & Buildings (\%) \\
\hline High & $0-1$ & 63 \\
\hline Medium & $1-2$ & 30 \\
\hline Low & +2 & 7 \\
\hline
\end{tabular}

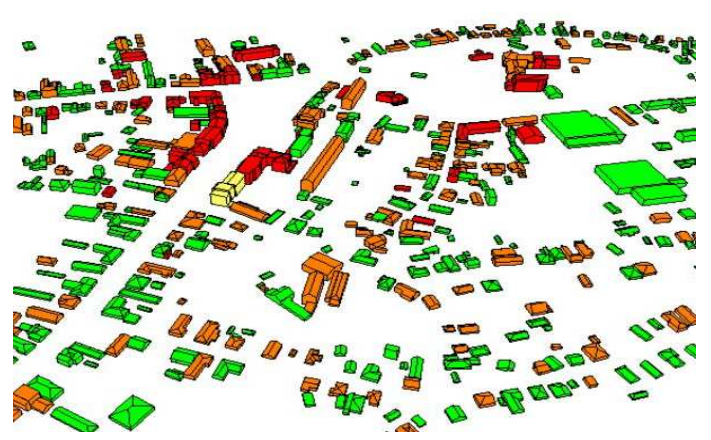

Figure 14. 3D building models displayed by the value of the standard deviation (green STDEV=0-1m, yellow STDEV=1$2 \mathrm{~m}$, red $\mathrm{STDEV}=+2 \mathrm{~m})$ 


\section{CONCLUSIONS}

The paper presented a research investigation for LOD2 building model generation using in-house and commercial tools. Research tools suffer of scalability and parameter generalization whereas the commercial tool needs very accurate and geolocalized data to achieve satisfactory $3 \mathrm{D}$ results.

There is still space of investigation and development in the $3 \mathrm{D}$ building model generation, in particular when using photogrammetric point clouds which are general more noisy than LiDAR data.

In the study case used for testing Building Reconstruction:

- $96 \%$ of buildings were reconstructed using cell decomposition

- $4 \%$ using extrusion.

$7 \%$ of the reconstructed buildings have a standard deviation higher than 2 meters and this is caused by the high complexity of the buildings or by the unreliable footprints.

Moreover the use of ancillary data (such as footprints) is still the most common approach, limiting (or affecting) the production of 3D geometries in case such data are not available.

\section{References}

Biljecki, F., Ledoux, H., Stoter, J., 2016. An improved LOD specification for 3D building models: Computers, Environment and Urban Systems.

Dorninger, P. \& Pfeifer, N., 2008. A Comprehensive Automated 3D Approach for Building Extraction, Reconstruction, and Regularization from Airborne Laser Scanning Point Clouds. Sensors. Vol. 8:11. pp. 7323-7343. DOI: $10.3390 / \mathrm{s} 8117323$. ISSN 1424-8220.

Gröger, G. \& Plümer, L., 2012. CityGML - Interoperable semantic 3D city models. ISPRS Journal of Photogrammetry and Remote Sensing. Vol. 71. pp 12-33. DOI: 10.1016/j.isprsjprs.2012.04.004. ISSN: 0924-2716.

Haala, N., Kada, M., 2010. An Update on Automatic 3D Building Reconstruction. Isprs Journal of Photogrammetry and Remote Sensing 65, 570-580.

Lafarge, F. \& Mallet, C., 2012. Creating Large-Scale City Models from 3D-Point Clouds: A Robust Approach with Hybrid Representation. International journal of computer vision 99, 69- 85 .

Nan, L., Wonka, P., 2017. PolyFit: Polygonal Surface Reconstruction from Point Clouds: Proceedings of the IEEE Conference on Computer Vision and Pattern Recognition. Venice, Italy, pp. 2353-2361.

Nex, F., Gerke, M., Remondino, F., Przybilla, H.-J., Bäumker, M., Zurhorst, A., 2015. ISPRS benchmark for multi-platform photogrammetry: ISPRS Annals of the Photogrammetry, Remote Sensing and Spatial Information Sciences, Vol. II3/W4, pp. 135-142.

Ozdemir, E., Remondino, F., 2018. Segmentation of 3D photogrammetric point cloud for 3D building modeling: ISPRS International archives of photogrammetry, remote sensing and spatial information sciences, Vol. XLII-4/W10, pp. 135-142.

Rabbani, T., van den Heuvel, F. A., Vosselman, G., 2006. Segmentation of point clouds using smoothness constraint: International archives of photogrammetry, remote sensing and spatial information sciences, Vol. 36(5).

Remondino, F., Gerke, M., 2015. Oblique Aerial Imagery - A Review: Proc. Photogrammetric Week 2015, D. Fritsch (Ed.), pp. $75-83$

Remondino, F., Toschi, I., Gerke, M., Nex, F., Holland, D., McGill, A., Talaya Lopez, J., Magarinos, A., 2016. Oblique Aerial Imagery for NMA - Some Best Practices: International archives of photogrammetry, remote sensing and spatial information sciences, Vol. XLI-B4.

\section{References from websites:}

Nan, L., 2018: https://3d.bk.tudelft.nl/liangliang/software.html (view at 10 June 2018).

Random sample consensus, 2018:

https://en.wikipedia.org/wiki/Random_sample_consensus (view at 17 June 2018).

REA Grant Agreement, 2017:

https://ec.europa.eu/research/participants/portal/desktop/en/opp ortunities/h2020/calls/h2020-msca-rise-

2018.html\#c,topics=callIdentifier/t/H2020-MSCA-RISE-2018 (view at 15 November 2017).

Ushakov, S., n.d.: http://pointclouds.org/documentation/ tutorials/region_growing_segmentation.php (view at 13 June 2018).

Virtual City System, 2018:

https://www.virtualcitysystems.de/en (view at 23 June 2018).

VOLTA, 2017: http://volta.fbk.eu (view at 5 March 2018). 